\title{
Contraceptive Patch
}

National Cancer Institute

\section{Source}

National Cancer Institute. Contraceptive Patch. NCI Thesaurus. Code C92760.

A dermal patch that contains estrogen and progestin hormones for release and absorption through the skin and into the body to prevent pregnancy. 